\section{BMJ Open} Ophthalmology

\title{
Validating e-norms methodology in ophthalmic biometry
}

\author{
H John Shammas (D) , Joe F Jabre ${ }^{2}$
}

To cite: Shammas HJ, Jabre JF. Validating enorms methodology in ophthalmic biometry. BMJ Open Ophthalmology 2020;5:e000500. doi:10.1136/ bmjophth-2020-000500

Received 3 May 2020 Revised 27 July 2020 Accepted 17 August 2020
Check for updates

\section{(c) Author(s) (or their} employer(s)) 2020. Re-use permitted under CC BY-NC. No commercial re-use. See rights and permissions. Published by BMJ

${ }^{1}$ Ophthalmology, USC Keck School of Medicine, Los Angeles, California, USA

${ }^{2}$ Neurology, David Geffen School of Medicine, Los Angeles, California, USA

Correspondence to Dr H John Shammas; hshammas@aol.com

\section{ABSTRACT}

Objective To validate the extrapolated norms or e-norms methodology in establishing a reference range for the biometric data used for intraocular lens power calculation.

Methods and Analysis All measurements were performed with an optical low-coherence reflectometer. A novel technique, the e-norms methodology, was used to determine the normative values of measurements. Results Eyes $(n=500)$ were measured to evaluate the axial length (AL), $\mathrm{K}$ readings (Ks), anterior chamber depth (ACD) and lens thickness (LT). Using the enorms methodology, the normal AL ranged from 22.50 to $24.50 \mathrm{~mm}$ (mean=23.50 $\mathrm{mm}$ ), with medium-long eyes between 24.51 and $24.99 \mathrm{~mm}$ and the long eyes measuring $25.00 \mathrm{~mm}$ and longer; the medium-short eyes ranged from 22.01 and $22.49 \mathrm{~mm}$, with the short eyes measuring $22.00 \mathrm{~mm}$ and shorter. Normal values ranged from 2.50 to $3.50 \mathrm{~mm}$ for $\mathrm{ACD}$ (mean=3.00 $\mathrm{mm}$ ), from 4.40 to $5.44 \mathrm{~mm}$ for $\mathrm{LT}$ (mean=4.92 $\mathrm{mm}$ ), and from 42.50 to 44.82 dioptres for Ks (mean=43.66 dioptres).

Conclusion Measurements of the biometric mean values compared favourably with published data. The e-norms methodology assisted in establishing a biometric reference range. Furthermore, it allowed us to cluster patients into groups based on AL differences.

\section{INTRODUCTION}

The extrapolated norms or e-norms methodology makes it possible to derive a cohort's 'normal' values from a laboratory population using a behaviour displayed by variables derived from normal individuals, one that distinguishes them from variables that are derived from individuals with pathology. ${ }^{1}$ This behaviour is referred to as "e-norms clustering', whereby the range of a laboratory variable obtained in normal individuals is smaller than the range of the laboratory variable obtained in abnormal individuals. Using this methodology, one can then identify and extract 'normal' values from patients' data sets by using this property of small versus large differences between successive values.

The e-norms method has been validated to date in a wide variety of neurophysiological applications. ${ }^{23}$ Our study represents the first attempt to use it in evaluating biometric ocular data. In this retrospective study, we evaluated the biometric data used for

\section{Key messages}

What is already known about this subject?

Normal values for biometric data used for intraocular power calculation vary according to different studies.

\section{What are the new findings?}

> A novel technique, the extrapolated norms or enorms method, is used to establish reference axial length, keratometry, anterior chamber depth and lens thickness values.

- The e-norms methodology is validated as a trusted method to calculate the mean values of ophthalmic measurements.

\section{How might these results change the focus of} research or clinical practice?

The e-norms methodology also allows the extraction of reference biometric values and cluster patients into groups based on axial length differences.

intraocular lens (IOL) power calculation, namely the axial length (AL), keratometric readings (Ks), anterior chamber depth (ACD) and lens thickness (LT) values, in a series of 500 consecutive cataractous eyes of 500 patients scheduled for cataract surgery, and we applied the e-norms methodology to extrapolate the likely normative values. To validate the new methodology in ophthalmic biometry, the results were compared with the results of four other studies from the same practice. $^{4-7}$

\section{MATERIALS AND METHODS}

\section{Study population and data collection}

This was a non-interventional study comprising a retrospective chart review of patients with a history of biometry examination prior to cataract surgery at one centre. A waiver of informed consent was granted to allow the use of de-identified patient data.

We analysed the measurements taken from 500 eyes of 500 patients scheduled to have cataract surgery between January 2017 and December 2017. All measurements were taken by the Lenstar LS 900 software (V.2.1.1; Haag-Streit). If both eyes of the patient 


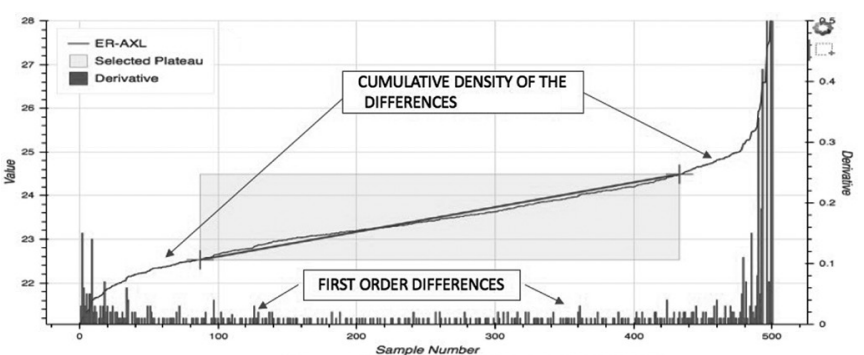

Figure 1 The e-norms analysis of the axial length measurements, derived from enorms.com. The first-order differences are represented by the vertical lines at the bottom of the graph, and the cumulative distribution of the variable by rank is represented by the inverted $\mathrm{S}$ curve. e-norms, extrapolated norms.

were operated on, only the first eye to be operated was included in the study.

\section{Measurement technique}

The device measures AL, ACD and LT using optical low-coherence reflectometry ${ }^{5}$ and calculates the $\mathrm{K}$ values using an integrated automated keratometer. In this paper, all $\mathrm{K}$ values refer to the average $\mathrm{K}$ ( $(\mathrm{K}$ flat $+\mathrm{K}$ steep) /2) given by the device using a 1.3375 keratometric index of refraction. The Lenstar LS 900 is calibrated daily before obtaining the measurements. At each session, the patient is asked to fixate on a flashing red light, and as soon as the image of the eye on the computer monitor is in focus a measurement is obtained. All measurements are repeated five consecutive times. The unit eliminates non-usable measurements. The average value is then recorded.

\section{The e-norms methodology}

We used the e-norms method ${ }^{8-10}$ to determine the normative values of the AL in this cohort study. In our study, 'normal' values refer to standard AL values that represent the common and usual type.

A Microsoft Excel spreadsheet containing the data to be analysed was uploaded anonymously and securely to an SSL (secure sockets layer) encrypted web application developed by one of the authors (JFJ). The e-norms application ${ }^{10}$ is first performed on the AL measurements:

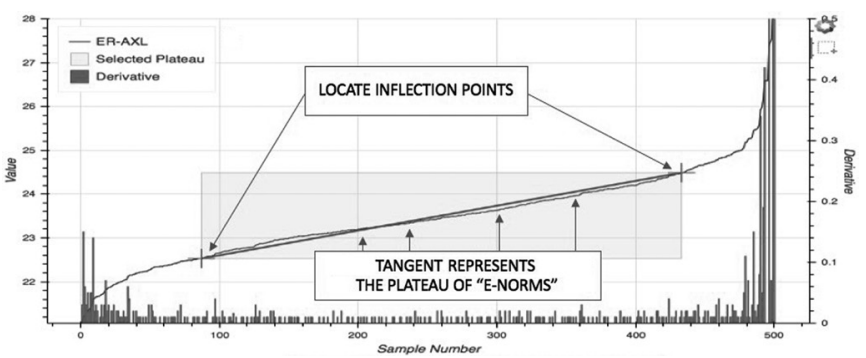

Figure 2 The e-norms analysis of the axial length measurements, derived from enorms.com. The inflection points are identified, and the straight fit line connecting the two inflection points represents the plateau of 'e-norms'. enorms, extrapolated norms.

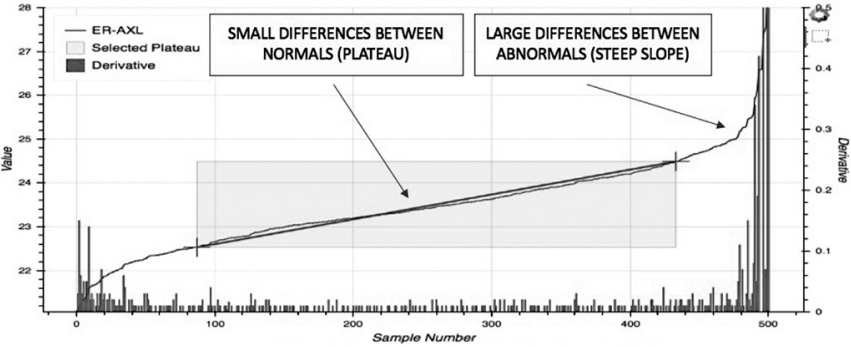

Figure 3 The e-norms analysis of the axial length measurements, derived from enorms.com. Note the small differences between the normal values within the 'plateau' revealing e-norms clustering, and the larger differences between the abnormal values represented by the steeper slope. e-norms, extrapolated norms.

- All measurements are sorted by ascending values: AL1, AL2, AL3, .....

- The first-order differences are calculated: AL2-AL1, AL3-AL2, ....

- The software creates a display (figure 1) showing the first-order differences on the bottom of the display. The data points at the left and right extremes show higher first-order differences between them, whereas those at the centre display smaller first-order differences, with steady increments.

- The software also displays a graph (figure 1) that represents the cumulative distribution of the variable by rank. The inverted $\mathrm{S}$ curve has a steep lower left, a middle 'plateau' and a steeper upper right.

- The inflection points are identified (figure 2); these represent the points where the steep lower left portion of the curve meets the middle plateau area on the left, and where the plateau portion of the curve changes into the steep upper portion of the curve. A straight fit line is drawn between the two points. This line represents the plateau of 'e-norms' (figure 2).

- Note that the plateau represents that special section of the data set with the low steady increments (figure 3), indicating that when examining the valueto-value differences of ranked continuous measurements, the plateau population can be characterised with the slowest rate of increase. These represent the normal values of the data being analysed. The graph also shows that the values in the lower left (short eyes) and upper right (long eyes) of the curve display larger first-order differences, making the slope steeper on both sides.

- The program evaluates the values within the plateau and calculates the mean of these values, SD, the minimal and maximal values, and the limits within $\pm 2 \mathrm{SD}$ of the mean.

The same analysis was conducted on the cases with an AL below the lower limits of the plateau to identify the medium-short eyes and the short eyes, and the cases exceeding the upper limits of the plateau to identify the medium-long eyes and the long eyes. The Ks, ACD and LT values were also evaluated. 


\begin{tabular}{|c|c|c|c|}
\hline Measurement & Mean \pm SD & Mean -2SD & Mean +2SD \\
\hline \multicolumn{4}{|l|}{ Axial length (in mm) } \\
\hline Normal & $23.50 \pm 0.50$ & 22.5 & 24.5 \\
\hline Medium-long & $24.75 \pm 0.13$ & 24.51 & 24.99 \\
\hline Medium-short & $22.25 \pm 0.12$ & 22.01 & 22.49 \\
\hline $\begin{array}{l}\text { Anterior chamber } \\
\text { depth (in } \mathrm{mm} \text { ) }\end{array}$ & $3.00 \pm 0.25$ & 2.5 & 3.5 \\
\hline $\begin{array}{l}\text { Lens thickness (in } \\
\mathrm{mm} \text { ) }\end{array}$ & $4.92 \pm 0.26$ & 4.4 & 5.44 \\
\hline $\begin{array}{l}\text { K readings (in } \\
\text { dioptres) }\end{array}$ & $43.66 \pm 0.58$ & 42.5 & 44.82 \\
\hline
\end{tabular}

e-norm, extrapolated norm.

\section{Patient involvement}

There was no patient participation in the study. Patients were not involved in the research project at any time, neither in the design of the study nor in the conduct of the study.

\section{RESULTS}

The mean age of the 500 patients was $77.52 \pm 7.90$ years (range 51-94 years). There was a fairly even distribution of men $(49 \%)$ and women $(51 \%)$ in the study group.

Table 1 shows the mean, SD and the limits of the e-norms analysis. Eyes with normal AL values $(n=353)$ ranged from 22.50 to $24.50 \mathrm{~mm}$, with a mean value of $23.50 \pm 0.50 \mathrm{~mm}$. Sixty-five eyes were longer than $24.50 \mathrm{~mm}$; an e-norms study on these 65 eyes further divided the group into medium-long eyes $(\mathrm{n}=40)$ with an AL ranging from 24.51 to $24.99 \mathrm{~mm}$, and long eyes $(n=25)$ with an $\mathrm{AL}$ of $25.00 \mathrm{~mm}$ and longer. Eighty-two eyes were shorter than $22.50 \mathrm{~mm}$; an e-norms study on these 82 eyes further divided the group into medium-short eyes $(n=52)$ with an AL ranging from 22.01 to $22.49 \mathrm{~mm}$, and short eyes $(\mathrm{n}=30)$ with an AL of $22.00 \mathrm{~mm}$ or less.

Normal values for the ACD $(\mathrm{n}=355)$ ranged from 2.50 to $3.50 \mathrm{~mm}$ (mean of $3.00 \pm 0.25 \mathrm{~mm}$ ) with 82 eyes shallower than $2.50 \mathrm{~mm}$ and 63 eyes deeper than $3.50 \mathrm{~mm}$.

Normal values for LT $(\mathrm{n}=343)$ ranged from 4.40 to $5.44 \mathrm{~mm}$ (mean of $4.92 \pm 0.26 \mathrm{~mm}$ ) with 78 eyes thinner than $4.40 \mathrm{~mm}$ and 79 eyes thicker than $5.44 \mathrm{~mm}$.

Normal values for the Ks $(\mathrm{n}=289)$ ranged from 42.50 to 44.82 dioptres (D) (mean of $43.66 \pm 0.58 \mathrm{~mm}$ ) with 111 eyes flatter than $42.50 \mathrm{D}$ and 100 eyes steeper than 44.82 D.

Table 2 compares the mean values from this study with the results of four other studies from the same practice where measurements were obtained with an immersion A-scan, ${ }^{4}$ optical low-coherence reflectometry, ${ }^{5}$ partial coherence interferometry $^{6}$ and swept-source optical coherence tomography. ${ }^{7}$

Table 3 compares the mean values in our cohort with e-norms (calculated from normal values) versus the conventional method (calculated from the entire data). The mean values and 95\% CI are almost identical; however, the range of normal values $($ mean $\pm 2 \mathrm{SD})$ is much wider with the conventional method.

\section{DISCUSSION}

The e-norms methodology allows the use of measurements taken from a database derived from a clinic's own patient population to produce normative values for any parameter in this database. The e-norms method has been validated to date in a wide variety of neurophysiological applications. ${ }^{1-3}$ In a just completed work ${ }^{8}$ comparing the reliability of visual e-norms plateau identification by different observers, 20 raters drawn from a diverse pool of hospital workers were asked to identify the e-norms plateau in 393 upper and 284 lower extremity nerve conduction studies while blinded to the test they were analysing. There was no significant difference between their findings by an inter-rater analysis of variance without replication testing.

In this work, we have derived a reference range for the biometric measurements of patients scheduled for cataract surgery. A normal AL has arbitrarily been set in some studies as a measurement between 22.0 and $26.0 \mathrm{~mm}$ with short eyes measuring below $22.0 \mathrm{~mm}$ and long eyes measuring above $26.0 \mathrm{~mm} .{ }^{11}{ }^{12}$ In other studies, the normal AL values were arbitrarily set between 22.5 and $24.5 \mathrm{~mm},{ }^{13}$ between 22.5 and $25.5 \mathrm{~mm},{ }^{14}$ and between 23.5 and $26.0 \mathrm{~mm} .{ }^{13}$ This lack of consistency affects the reported accuracy of IOL power formulas in the so-called short, normal and long eyes. ${ }^{15}$ The e-norms methodology established five categories of AL based on the measured value: short eyes $(22.0 \mathrm{~mm}$ and less), medium-short $(22.01-22.49 \mathrm{~mm})$, average $(22.50-24.50 \mathrm{~mm})$, mediumlong (24.51-24.99 $\mathrm{mm})$ and long $(25.00 \mathrm{~mm}$ and over $)$.

\begin{tabular}{lccccc}
\hline Table 2 & Mean values as measured by different biometers & & & \\
\hline Measurements & Present & A-scan & OLCR & PCI & SS-OCT \\
\hline Axial length (in mm) & 23.5 & 23.46 & 23.55 & 23.3 & 23.35 \\
Anterior chamber depth (in mm) & 3 & 2.96 & 3.15 & 2.85 & N/A \\
Lens thickness (in mm) & 4.92 & 4.93 & 4.6 & 4.7 \\
K readings (in dioptres) & 43.66 & N/A & 43.81 & 43.79 & 43.87 \\
\hline
\end{tabular}

N/A, not applicable; OLCR, optical low-coherence reflectometry; PCI, partial coherence interferometrySS-OCT, swept-source optical coherence tomography. 
Table 3 Calculated values with e-norms versus conventional method in our cohort

\begin{tabular}{|c|c|c|c|c|}
\hline & \multicolumn{2}{|c|}{ E-norms method } & \multicolumn{2}{|c|}{ Conventional method } \\
\hline & Mean $(95 \% \mathrm{Cl})$ & Range & Mean $(95 \% \mathrm{Cl})$ & Range \\
\hline $\mathrm{AL}$ (in mm) & 23.50 (23.4 to 23.6 ) & $22.50-24.50$ & 23.49 (23.4 to 23.6$)$ & 21.33-25.66 \\
\hline ACD (in mm) & 3.00 (2.97 to 3.03$)$ & $2.50-3.50$ & 2.97 (2.93 to 3.01 ) & $2.05-3.89$ \\
\hline LT (in mm) & 4.92 (4.89 to 4.95$)$ & $4.40-5.44$ & 4.93 (4.88 to 4.98$)$ & $3.81-6.05$ \\
\hline Ks (in dioptres) & 43.66 (43.0 to 44.3$)$ & $42.50-44.82$ & 43.62 (43.5 to 43.8$)$ & $40.54-46.70$ \\
\hline
\end{tabular}

Range: mean $\pm 2 S D$.

$\mathrm{ACD}$, anterior chamber depth; AL, axial length; e-norms, extrapolated norms; Ks, keratometric readings; LT, lens thickness.

Our study represents the first attempt to use the e-norms methodology in evaluating biometric data. To validate our findings, the mean values derived in our study were compared with other biometric mean values derived from conventional methods where the entire data are used to calculate the mean values. Our results compared favourably with four other studies from the same practice (table 2). The A-scan study ${ }^{4}$ included 750 eyes and showed comparable results with the ones reported this study. The three other studies ${ }^{5-7}$ were based on smaller number of cases and showed minor variations from the present study. The major advantage of the e-norms methodology is that it allowed us to calculate the mean values from typically normal eyes and not from the entire group.

The e-norms mean values in our cohort were also compared with the calculated means by a conventional method on the same series (table 3). The mean values and $95 \% \mathrm{CI}$ are almost identical, further validating the e-norms methodology as a trusted method to calculate mean values. However, the conventional method yielded a much wider range of values, making it not suitable to calculate normal ranges.

An additional advantage of our method is the possibility of applying it successfully to smaller numbers within the cohort and subdividing certain groups. In our study, we applied the e-norms method on the longer eyes $(\mathrm{n}=65)$, further dividing them into medium-long with 40 eyes ranging from 24.51 to $24.99 \mathrm{~mm}$ and long with 25 eyes measuring $25.00 \mathrm{~mm}$ or longer. We then applied the e-norms method on the shorter eyes $(n=82)$ and further divided them into medium-short with 52 eyes ranging from 22.01 to $22.49 \mathrm{~mm}$ and short with 30 eyes measuring $22.00 \mathrm{~mm}$ or shorter.

Many research studies can be expensive and timeconsuming to complete. We believe that the e-norms methodology solves a problem that exists in any investigative research field where normative data that pertain to a subject cohort are needed. As a result, our method can be used on any cohort data; in our study, we elected to evaluate the biometric data of patients undergoing cataract surgery at our centre and establish five subgroups based on the AL differences. The method is fast and can be done using a local Excel spreadsheet or on the web using proper ethics permission.
In conclusion, we here described a new method to calculate normal biometric values quickly and easily. The speed and ease with which it can be accomplished are a major draw to use it in ophthalmology. In evaluating the biometric measurements in eyes undergoing cataract surgery, it allowed us to calculate the respective means, establish normative reference values and cluster patients into groups and subgroups based on AL differences.

Contributors JHS and JFJ participated in the concept and design of the study. JHS performed the measurements, collected the data and drafted the manuscript. JFJ is the author of the e-norms methodology. He analysed the data. JHS and JFJ interpreted the data and approved the manuscript.

Funding The authors have not declared a specific grant for this research from any funding agency in the public, commercial or not-for-profit sectors.

Competing interests None declared.

Patient and public involvement Patients and/or the public were not involved in the design, or conduct, or reporting, or dissemination plans of this research.

Patient consent for publication Not required.

Ethics approval The study was approved by the Milkie-Shammas Surgery Center Institutional Review Board (Lynwood, California). Data were collected in a manner consistent with the tenets of the Declaration of Helsinki.

Provenance and peer review Not commissioned; externally peer reviewed.

Open access This is an open access article distributed in accordance with the Creative Commons Attribution Non Commercial (CC BY-NC 4.0) license, which permits others to distribute, remix, adapt, build upon this work non-commercially, and license their derivative works on different terms, provided the original work is properly cited, appropriate credit is given, any changes made indicated, and the use is non-commercial. See: http://creativecommons.org/licenses/by-nc/4.0/.

ORCID iD

H John Shammas http://orcid.org/0000-0002-9307-5591

\section{REFERENCES}

1 Jabre JF, Pitt MC, Deeb J, et al. E-norms: a method to extrapolate reference values from a laboratory population. $J$ Clin Neurophysiol 2015;32:265-70.

2 Pitt MC, Jabre J. The problem of lack of normative data in paediatric EMG and possible solutions. Clin Neurophysiol 2018;129:672-5.

3 Jabre JF. Do you define the limits of normalcy from looking at the patient or the healthy subject? - An e-norms reply. Clin Neurophysiol 2018;129:1517-8.

4 Jivrajka R, Shammas MC, Boenzi T, et al. Variability of axial length, anterior chamber depth, and lens thickness in the cataractous eye. $J$ Cataract Refract Surg 2008;34:289-94.

5 Shammas HJ, Hoffer KJ. Repeatability and reproducibility of biometry and keratometry measurements using a noncontact optical low-coherence reflectometer and keratometer. Am J Ophthalmol 2012;153:55-61.

6 Shammas HJ, Chan S. Precision of biometry, keratometry, and refractive measurements with a partial coherence interferometrykeratometry device. J Cataract Refract Surg 2010;36:1474-8. 
7 Shammas HJ, Ortiz S, Shammas MC, et al. Biometry measurements using a new large-coherence-length swept-source optical coherence tomographer. J Cataract Refract Surg 2016;42:50-61.

8 Earle NE, Jabre JF. Reliability and reproducibility of visual e-norms plateau identification. Clin Neurophysiol Pract. In Press 2020;5:83-6.

9 Jabre JF. Proceedings of the 30 th international Congress of clinical neurophysiology. Berlin, Germany, 2014

10 Jabre J. Enorms, 2020. Available: https://enorms.com/

11 Hoffer KJ. The Hoffer Q formula: a comparison of theoretic and regression formulas. J Cataract Refract Surg 1993;19:700-12.
12 Cooke DL, Cooke TL. A comparison of two methods to calculate axial length. J Cataract Refract Surg 2019;45:284-92.

13 Barrett GD. An improved universal theoretical formula for intraocular lens power prediction. J Cataract Refract Surg 1993;19:713-20.

14 Melles RB, Holladay JT, Chang WJ. Accuracy of intraocular lens calculation formulas. Ophthalmology 2018;125:169-78.

15 Kane JX, Van Heerden A, Atik A, et al. Intraocular lens power formula accuracy: comparison of 7 formulas. J Cataract Refract Surg 2016;42:1490-500. 